\title{
Comparative studies of the magnetic dipole and electric quadrupole hyperfine constants for the ground and low lying excited states of ${ }^{25} \mathrm{Mg}^{+}$
}

\author{
Chiranjib Sur, Bijaya K. Sahoo, Rajat K Chaudhuri, B. P. Das \\ Non-Accelerator Particle Physics Group, Indian Institute of Astrophysics, \\ Bangalore - 560 034, India \\ D. Mukherjee \\ Indian Association for the Cultivation of Science, Kolkata - 700 032, India
}

(Received date, Accepted date)

\begin{abstract}
We have employed the relativistic coupled cluster theory to calculate the magnetic dipole and electric quadrupole hyperfine constants for the ground and low lying excited states of singly ionized magnesium. Comparison with experimental and the other theoretical results are done and predictions are also made for a few low lying excited states which could be of interest. We have made comparative studies of the important many body effects contributing to the hyperfine constants for the different states of the ion.

PACS number(s). : 31.15.Ar, 31.15.Dv, 31.25.Jf, 32.10.Fn
\end{abstract}

\section{Introduction}

The hyperfine interactions in alkali metal atoms and alkaline earth ions have been of interest for quite a long time [1, 2]. A number of theoretical investigations including some based on relativistic manybody theory have been performed [3, 4, 5] and they compare reasonably well with experiments. Some of the theoretically predicted values could be of experimental interest with the advent of high precision techniques [6] involving trapped and laser cooled atoms [7] and ions [2].

The high precision calculations of different properties of many-electron atoms requires accurate wavefunctions in the nuclear region as well as the region far from the nucleus. The study of properties like hyperfine constants requires the former. Since hyperfine interactions are sensitive to electron correlations, the determination of atomic hyperfine constant provides an important test for $a b$ initio atomic structure theory [8].

In this paper we have carried out ab-initio calculations of the magnetic dipole and electric quadrupole hyperfine constants and compared the many-body effects for the ground as well as some excited states. Section 2 provides the necessary theoretical background to the magnetic dipole $(A)$ and the electric quadrupole $(B)$ hyperfine constants. Section 3 gives an overview of the coupled-cluster theory and its application to this specific problem. Finally in section 4 the results of our calculations are presented and discussed.

\section{Theory}

The interaction between the various moments of the nucleus and the electrons of an atom are collectively referred to as hyperfine interactions [8]. In this paper we shall consider the interactions between the atomic electrons with the nuclear magnetic dipole and electric quadrupole moments. Nuclear spin gives rise to a nuclear magnetic dipole moment and the departure from spherical charge distribution in the nucleus produces an electric quadrupole moment.

The hyperfine interaction is given by 9

$$
H_{h f s}=\sum_{k} M^{(k)} \cdot T^{(k)}
$$


where $M^{(k)}$ and $T^{(k)}$ are spherical tensors of rank $k$, which corresponds to nuclear and electronic parts of the interaction respectively. The lowest $k=0$ order represents the interaction of the electron with the spherical part of the nuclear charge distribution. The eigenstates of the atomic Hamiltonian in the presence of a hyperfine interaction are denoted by $\left|I J F M_{F}\right\rangle$. Here $\mathbf{I}$ and $\mathbf{J}$ are the total angular angular momentum for the nucleus and the electron state, respectively, and $\mathbf{F}=\mathbf{I}+\mathbf{J}$ with the projection $M_{F}$.

In the first order perturbation theory, the energy corresponding to the hyperfine interaction of the fine structure state $\left|J M_{J}\right\rangle$ are the expectation values of $H_{h f s}$ such that

$$
\begin{aligned}
W(J) & =\frac{\left\langle I J F M_{F}\left|\sum_{k} M^{(k)} \cdot T^{(k)}\right| I J F M_{F}\right\rangle}{} \\
& =\sum_{k}(-1)^{I+J+F}\left\{\begin{array}{ccc}
I & J & F \\
J & I & k
\end{array}\right\}\left\langle I\left\|M^{(k)}\right\| I\right\rangle\left\langle J\left\|T^{(k)}\right\| J\right\rangle
\end{aligned}
$$

The $k=1$ term describes the magnetic dipole coupling of the nuclear magnetic moment with the magnetic field created by the electron at the position of the nucleus. The nuclear dipole moment $\mu_{I}$ is defined (in units of Bohr magneton $\mu_{N}$ ) as

$$
\mu_{I} \mu_{N}=\left\langle I I\left|M_{0}^{(1)}\right| I I\right\rangle=\left(\begin{array}{ccc}
I & 1 & I \\
-I & 0 & I
\end{array}\right)\left\langle I\left\|M^{(1)}\right\| I\right\rangle
$$

and the operator $T_{q}^{(1)}$ is given by[10]

$$
T_{q}^{(1)}=\sum_{q} t_{q}^{(1)}=\sum_{j}-i e \sqrt{\frac{8 \pi}{3}} r_{j}^{-2} \overrightarrow{\alpha_{j}} \cdot \mathbf{Y}_{1 q}^{(0)}\left(\widehat{r}_{j}\right) .
$$

Here $\vec{\alpha}$ is the Dirac matrix and $\mathbf{Y}_{k q}^{\lambda}$ is the vector spherical harmonics. In Eq. (4) the index $j$ refers to the $j$-th electron of the atom and $e$ is the magnitude of the electronic charge. The magnetic dipole hyperfine constant $A$ is defined as

$$
A=\mu_{N}\left(\frac{\mu_{I}}{I}\right) \frac{\left\langle J\left\|T^{(1)}\right\| J\right\rangle}{\sqrt{J(J+1)(2 J+1)}},
$$

and the corresponding magnetic dipole hyperfine energy $W_{M 1}$ is given by

$$
W_{M 1}=A\langle I \cdot J\rangle=A \frac{K}{2},
$$

where $K=F(F+1)-I(I+1)-J(J+1)$.

The second order term in the hyperfine interaction is the electric quadrupole part. The electric quadrupole hyperfine constant is defined by putting $k=2$ in Eq. (2). The nuclear quadrupole moment is defined as

$$
T_{q}^{(2)}=\sum_{q} t_{q}^{(2)}=\sum_{j}-e r_{j}^{-3} C_{q}^{(2)}\left(\widehat{r_{j}}\right),
$$

Here, $C_{q}^{(k)}=\sqrt{\frac{4 \pi}{(2 k+1)}} Y_{k q}$, with $Y_{k q}$ being the spherical harmonic. Hence the electric quadrupole hyperfine constant $B$ is

$$
B=2 e Q\left[\frac{2 J(2 J-1)}{(2 J+1)(2 J+2)(2 J+3)}\right]^{1 / 2}\left\langle J\left\|T^{(2)}\right\| J\right\rangle,
$$

and the corresponding electric quadrupole hyperfine energy $W_{E 2}$ is given by

$$
W_{E 2}=\frac{B}{2} \frac{3 K(K+1)-4 I(I+1) J(J+1)}{2 I(2 I-1) 2 J(2 J-1)} .
$$

In Eq. (4)and 7$) t_{q}^{(k)}$ are the single particle reduced matrix element for the electronic part. The reductions of the single particle matrix elements into angular factors and radial integral are straightforward by means of using the Wigner Eckart theorem. These single particle reduced matrix elements are given by

$$
\left\langle\kappa\left\|t_{q}^{(1)}\right\| \kappa^{\prime}\right\rangle=-\left\langle\kappa\left\|C_{q}^{(1)}\right\| \kappa^{\prime}\right\rangle\left(\kappa+\kappa^{\prime}\right) \int d r \frac{\left(P_{\kappa} Q_{\kappa^{\prime}}+Q_{\kappa} P_{\kappa^{\prime}}\right)}{r^{2}}
$$

and 


$$
\left\langle\kappa\left\|t_{q}^{(2)}\right\| \kappa^{\prime}\right\rangle=-\left\langle\kappa\left\|C_{q}^{(2)}\right\| \kappa^{\prime}\right\rangle \int d r \frac{\left(P_{\kappa} P_{\kappa^{\prime}}+Q_{\kappa} Q_{\kappa^{\prime}}\right)}{r^{3}},
$$

where $\left\langle\kappa\left\|C_{q}^{(k)}\right\| \kappa^{\prime}\right\rangle$ is the reduced matrix element of the spherical tensor and is equal to

$$
(-1)^{j+1 / 2} \sqrt{(2 j+1)\left(2 j^{\prime}+1\right)}\left(\begin{array}{ccc}
j & k & j^{\prime} \\
\frac{1}{2} & 0 & -\frac{1}{2}
\end{array}\right) \pi\left(l, k, l^{\prime}\right)
$$

with

$$
\pi\left(l, k, l^{\prime}\right)=\left\{\begin{array}{cc}
1 & \text { if } l+k+l^{\prime} \text { even } \\
0 & \text { otherwise }
\end{array}\right.
$$

Here the single particle orbitals are expressed in terms of the Dirac spinors with $P_{i}$ and $Q_{i}$ as large and small components respectively.

\section{Overview of the coupled cluster theory : method of calculation}

We start with an $N$ electron closed shell Dirac-Fock (DF) reference state $|\Phi\rangle$. The corresponding correlated closed shell state is then

$$
|\Psi\rangle=\exp (T)|\Phi\rangle,
$$

where $T$ is the core electron excitation operator. Then the Dirac-Coulomb eigenvalue equation is

$$
H \exp (T)|\Phi\rangle=E \exp (T)|\Phi\rangle,
$$

with the Dirac-Coulomb Hamiltonian

$$
H=\sum_{i}\left(c \alpha_{i} \cdot p_{i}+\left(\beta_{i}-1\right) m c^{2}+V_{N}\right)+\sum_{i<j} \frac{1}{r_{i j}} .
$$

This leads to the exact ground state energy $E$ of the closed-shell part of the system. Here $\alpha_{i}$ and $\beta_{i}$ are Dirac matrices and $V_{N}$ is the nuclear potential. If we consider the DF state $|\Phi\rangle$ as the Fermi vacuum, then the normal ordered Hamiltonian is

$$
H_{N} \equiv H-\langle\Phi|H| \Phi\rangle=H-E_{D F} .
$$

If we project $\langle\Phi| \exp (-T)$ from the left we obtain the correlation energy $(\Delta E)$ and if we project any of the excited determinant $\left\langle\Phi^{\star}\right| \exp (-T)$ we additionally get a set of equations which are used to obtain the $T$ amplitudes. Using the normal ordered dressed Hamiltonian $\overline{H_{N}}=\exp (-T) H_{N} \exp (T)$ the corresponding equations for correlation energy and amplitudes become

$$
\left\langle\Phi\left|\overline{H_{N}}\right| \Phi\right\rangle=\Delta E,
$$

and

$$
\left\langle\Phi^{\star}\left|\overline{H_{N}}\right| \Phi\right\rangle=0 .
$$

Here the state $\left|\Phi^{\star}\right\rangle$ may be singly excited $\left|\Phi_{a}^{r}\right\rangle$ or double excited $\left|\Phi_{a b}^{r s}\right\rangle$ and so on. The indices $a, b, \cdots$ refer to holes and $p, q, \cdots$ to particles. We have considered the coupled cluster single and double (CCSD) approximation, where the cluster operator $T$ is composed of one- and two-body excitation operators, i.e. $T=T_{1}+T_{2}$, and are expressed in second quantization form

$$
T=T_{1}+T_{2}=\sum_{a p} a_{p}^{\dagger} a_{a} t_{a}^{p}+\frac{1}{2} \sum_{a b p q} a_{p}^{\dagger} a_{q}^{\dagger} a_{b} a_{a} t_{a b}^{p q} .
$$

Contracting the ladder operators [11] and rearranging the indices, the amplitude equations can be expressed in the form

$$
A+B(T) \cdot T=0,
$$


where $A$ is a constant vector consisting of the matrix elements $\left\langle\Phi^{\star}\left|H_{N}\right| \Phi\right\rangle, T$ is the vector of the excitation amplitudes and $B(T)$ is the matrix which depends on the cluster amplitudes itself so that Eq. (19) is solved self-consistently. For example, a typical contribution to the term $\widehat{H_{N} T_{2}} T_{2}$ is

$$
B_{a b}^{p q}=\frac{1}{2} \sum_{d g r s} V_{d g r s} t_{a d}^{p r} t_{g b}^{s q}
$$

Here $V_{d g r s}$ is the two-electron Coulomb integral and $t_{a d}^{p r}$ is the cluster amplitude corresponding to a simultaneous excitation of two electrons from orbital $a$ and $d$ to $p$ and $r$ respectively. To obtain a full set of terms which contribute to this specific excitation, diagrammatic techniques are used.

The ground state of ${ }^{25} \mathrm{Mg}^{+}$contains only one valance electron in the outer most orbital $\left(3 s_{1 / 2}\right)$. To calculate the ground state energy of the system we first compute the correlations for the closed shell system $\left({ }^{25} \mathrm{Mg}^{+2}\right)$ using the closed shell coupled cluster approach and then use the technique of electron attachment (open shell coupled cluster (OSCC)) method. The energy of the excited state are obtained by the same way. In order to add an electron to the $k$ th virtual orbital of the DF reference state we define

$$
\left|\Phi_{k}^{N+1}\right\rangle \equiv a_{k}^{\dagger}|\Phi\rangle
$$

with the particle creation operator $a_{k}^{\dagger}$. Then by using the excitation operators for both the core and valance electron the exact state is defined as [8]:

$$
\left|\Psi_{k}^{N+1}\right\rangle=\exp (T)\left\{\exp \left(S_{k}\right)\right\}\left|\Phi_{k}^{N+1}\right\rangle .
$$

Here $\left\{\exp \left(S_{k}\right)\right\}$ is the normal ordered exponential representing the valance part of the wave operator. Here

$$
S_{k}=S_{1 k}+S_{2 k}=\sum_{k \neq p} a_{p}^{\dagger} a_{k} s_{k}^{p}+\frac{1}{2} \sum_{b p q} a_{p}^{\dagger} a_{q}^{\dagger} a_{b} a_{k} s_{k b}^{p q},
$$

where $k$ stands for valance orbital. $S_{k}$ contain the particle annihilation operator $a_{k}$, and because of the normal ordering it cannot be connected to any other valance electron excitation operator and then $\left\{\exp \left(S_{k}\right)\right\}$ automatically reduces to $\left\{1+S_{k}\right\}$.

Then we can write the Eq.(22) as

$$
\left|\Psi_{k}^{N+1}\right\rangle=\exp (T)\left\{1+S_{k}\right\}\left|\Phi_{k}^{N+1}\right\rangle .
$$

Following the same procedure as in the closed-shell approach, we obtain a set of equations

$$
\left\langle\Phi_{k}^{N+1}\left|\overline{H_{N}}\left\{1+S_{k}\right\}\right| \Phi_{k}^{N+1}\right\rangle=H_{e f f}
$$

and

$$
\left\langle\Phi_{k}^{{ }^{*} N+1}\left|\overline{H_{N}}\left\{1+S_{k}\right\}\right| \Phi_{k}^{N+1}\right\rangle=H_{\text {eff }}\left\langle\Phi_{k}^{{ }^{*} N+1}\left|\left\{1+S_{k}\right\}\right| \Phi_{k}^{N+1}\right\rangle,
$$

where the desired roots can be obtained by diagonalizing $H_{e f f}$. The Eq.(26) is non-linear in $S_{k}$ because the energy difference $H_{\text {eff }}$ is itself a function of $S_{k}$. Hence, these equations have to solved self-consistently to determine the $S_{k}$ amplitudes.

Triple excitations are included in our open shell $\mathrm{CC}$ amplitude calculations by an approximation that is similar in spirit to $\operatorname{CCSD}(\mathrm{T})$ [12. The approximate triple excitation amplitude is given by

$$
S_{a b k}^{p q r}=\frac{\widehat{V T_{2}}+\widehat{V S_{2}}}{\varepsilon_{a}+\varepsilon_{b}+\varepsilon_{k}-\varepsilon_{p}-\varepsilon_{q}-\varepsilon_{r}},
$$

where $S_{a b k}^{p q r}$ are the amplitudes corresponding to the simultaneous excitation of orbitals $a, b, k$ to $p, q, r$ respectively and $\widehat{V T}$ and $\widehat{V S}$ are the correlated composites involving $V$ and $T$, and $V$ and $S$ respectively. $\varepsilon_{k}$ is the orbital energy of the $k$ th orbital. The above amplitudes (some representative diagrams are given in figure 11) are added appropriately in the singles and doubles open shell cluster amplitude equations and these equations are then solved self-consistently. We therefore obtain solutions of $S_{1}$ and $S_{2}$ amplitudes taking into consideration the effect of the triple excitations in an approximate way

The expectation value of any operator $O$ can be written as the normalized form with respect to the exact state $\left|\Psi^{N+1}\right\rangle$ as 


$$
\langle O\rangle=\frac{\left\langle\Psi^{N+1}|O| \Psi^{N+1}\right\rangle}{\left\langle\Psi^{N+1} \mid \Psi^{N+1}\right\rangle}=\frac{\left\langle\Phi^{N+1}\left|\left\{1+S^{\dagger}\right\} \exp \left(T^{\dagger}\right) O \exp (T)\{1+S\}\right| \Phi^{N+1}\right\rangle}{\left\langle\Phi^{N+1}\left|\left\{1+S^{\dagger}\right\} \exp \left(T^{\dagger}\right) \exp (T)\{1+S\}\right| \Phi^{N+1}\right\rangle} .
$$

For computational simplicity we store only the one-body matrix element of $\bar{O}=\exp \left(T^{\dagger}\right) O \exp (T) . \bar{O}$ may be expressed in terms of uncontracted single-particle lines [13. The fully contracted part of $\bar{O}$ will not contribute as it cannot be linked with the remaining part of the numerator of the above equation.

In the LCCSD approximation Eq. (22) turns out to be

$$
\left|\Psi_{k}^{N+1}\right\rangle=\left\{1+T+S_{k}\right\}\left|\Phi_{k}^{N+1}\right\rangle,
$$

and $\overline{H_{N}}=H_{N}+\overbrace{H_{N} T}$.

The closed and open shell cluster amplitude equations reduce to

$$
\left\langle\Phi_{0}^{k}|H_{N}+\overbrace{H_{N} T}| \Phi_{0}\right\rangle=0,
$$

and

$$
\left\langle\Phi_{k}^{\star} N+1\left|\overline{H_{N}}\left\{1+S_{k}\right\}\right| \Phi_{k}^{N+1}\right\rangle=\left\langle\Phi_{k}^{\star} N+1\left|S_{v}\right| \Phi_{k}^{N+1}\right\rangle\left\langle\Phi_{k}^{N+1}\left|H_{N}\right| \Phi_{k}^{N+1}\right\rangle .
$$

The orbitals used in the present work are expanded in terms of a finite basis set comprising of Gaussian type orbitals (GTO) 14

$$
F_{i, k}(r)=r^{k} \exp \left(-\alpha_{i} r^{2}\right),
$$

with $k=0,1,2 \cdots$ for $s, p, d, \cdots$ type functions, respectively. The exponents are determined by the even tempering condition [15]

$$
\alpha_{i}=\alpha_{0} \beta^{i-1} .
$$

The staring point of the computation is the generation of the Dirac-Fock (DF) orbitals [14] which are defined on a radial grid of the form

$$
r_{i}=r_{0}[\exp (i-1) h-1]
$$

with the freedom of choosing the parameters $r_{0}$ and $h$. All DF orbitals are generated using a two parameter Fermi nuclear distribution

$$
\rho=\frac{\rho_{0}}{1+\exp ((r-c) / a)},
$$

where the parameter $c$ is the half charge radius and $a$ is related to skin thickness, defined as the interval of the nuclear thickness in which the nuclear charge density falls from near one to near zero.

Although we have used a large basis for the generation of the single particle orbitals, the high-lying virtual orbitals (above a certain threshold) are kept frozen as their contributions to the high-lying virtuals in the $T$ and $S$ amplitudes in the CC equations are negligible. Another advantage of this approximation is that it reduces the memory required to store the matrix elements of the dressed operator $\bar{H}$ and the two-electron Coulomb integrals in the main memory, thereby reducing the computational cost. In our calculations, we have included all possible single, double and partial triple excitations from the core.

We have used different $\alpha_{0}$ and $\beta$ for different symmetries. The number of basis functions used to generate the even tempered DF states are listed in table 1 and the values of the parameters $\alpha_{0}$ and $\beta$ used are also listed. For coupled cluster calculations, we have restricted the basis by imposing an upper bound in energy for single particle orbitals and the convergence of our results are shown in table 3 .

\section{Results and discussions}

The values of the magnetic dipole hyperfine constant $A$ and electric quadrupole hyperfine constant $B$ for different states are given in table 2. Our calculated value of $A$ for the ground state $3 s_{1 / 2}$ is in good agreement (less than $0.6 \%$ ) with experiment and it is more accurate than a previous calculation $(\sim 1 \%)$ based on second order relativistic many-body perturbation theory (RMBPT) [3]. This is because unlike the previous work our calculation is based on an approach which is equivalent to all-order MBPT. In particular, we have taken into account all single, double and a subset of triple excitations to all orders in the residual Coulomb interaction. It is, therefore, not surprising that the result of our calculation when 


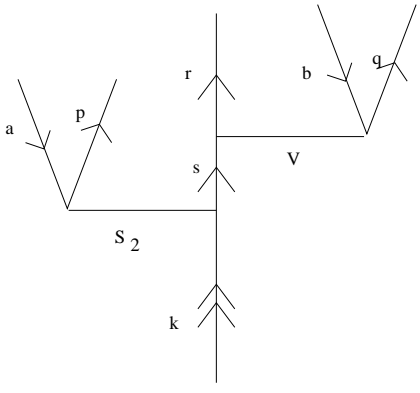

(a)

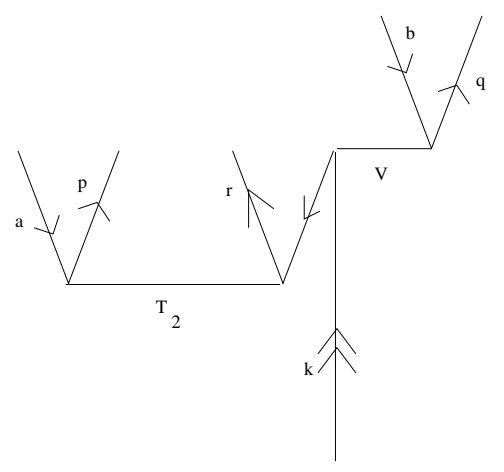

(b)

Figure 1: Some typical important diagrams which arise due to the inclusion of triples through Eq. 27)
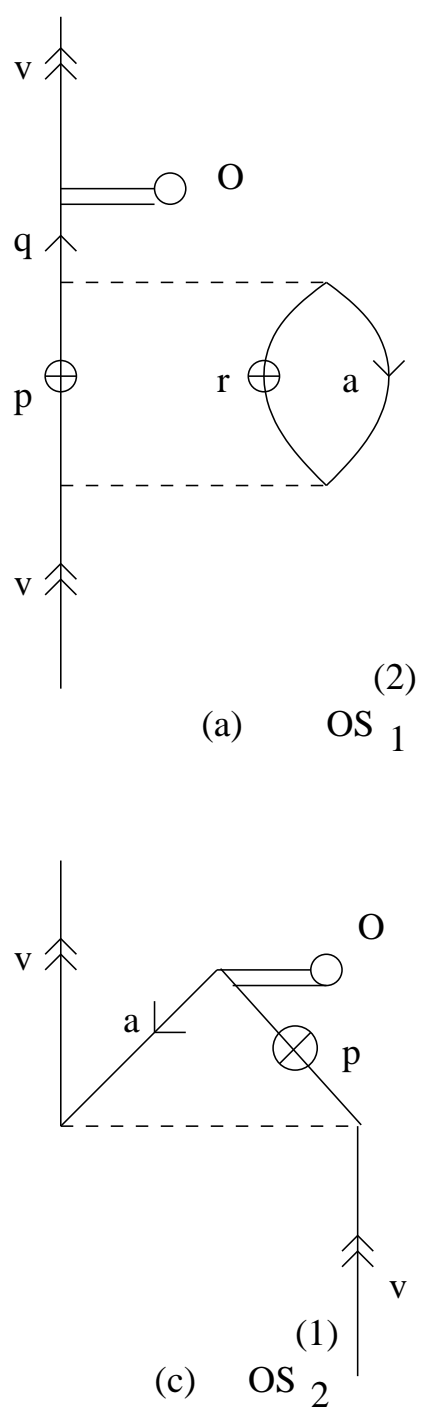

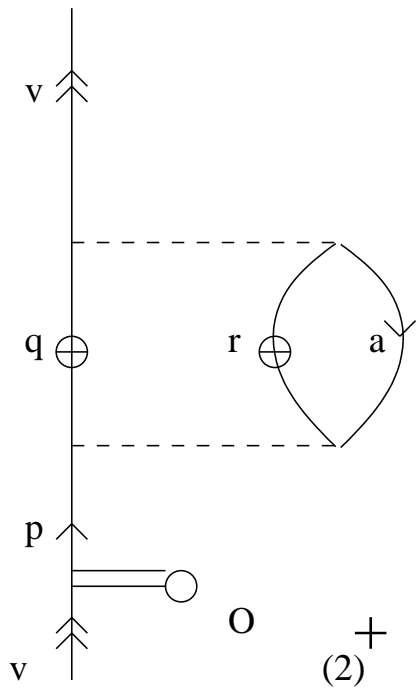

(b) $\mathrm{S}_{1} \mathrm{O}$

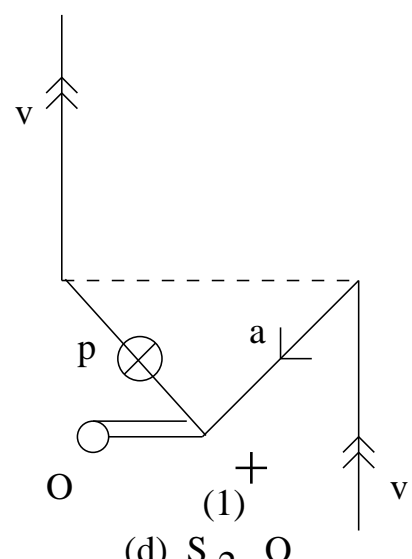

(d) $\mathrm{S}_{2} \mathrm{O}$

Figure 2: Some typical important MBPT diagrams for pair correlation and core-polarization effects. The superscripts refer to the order of perturbation and the dashed lines correspond to the Coulomb interaction. Particles and holes (labeled by $a$ ) are denoted by the lines directed upward and downward respectively. The double line represents the $\mathrm{O}$ (the hyperfine interaction operator) vertices. The valance (labeled by $v$ ) and virtual orbitals (labeled by $p, q, r .$. ) are depicted by double arrow and single arrow respectively, whereas the orbitals denoted by $\oplus$ can either be valance or virtual. 


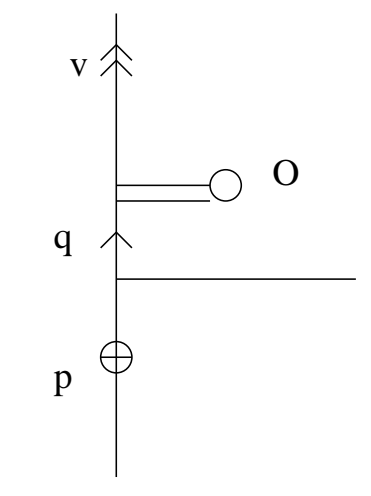

(a) $\mathrm{OS}_{1}$

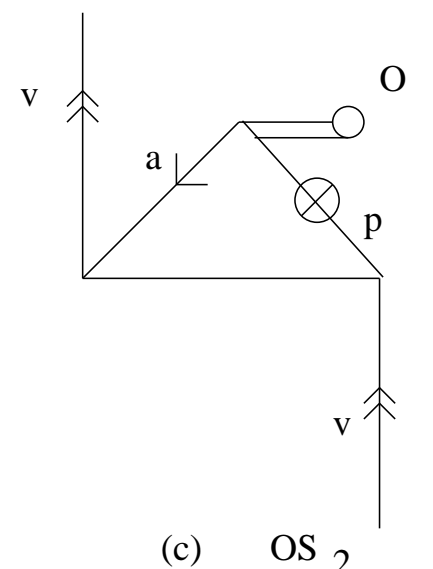

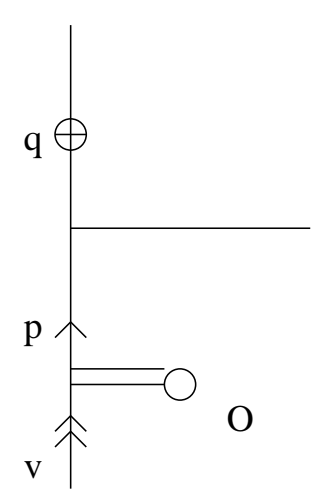

(b) $\mathrm{S}_{1}^{+} \mathrm{O}$

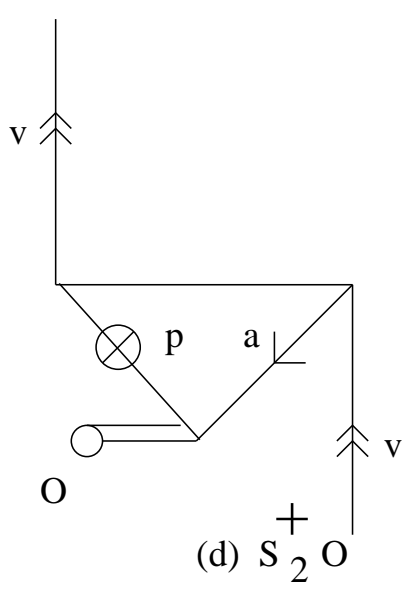

Figure 3: The corresponding CC diagrams for pair correlation and core-polarization effects. Here $a$ denotes a hole whereas $v$ denotes valance orbital and $p, q, r \ldots$ denote virtual orbitals (particles). 
Table 1: No. of basis functions used to generate the even tempered Dirac-Fock orbitals and the corresponding value of $\alpha_{0}$ and $\beta$ used.

\begin{tabular}{cccccccc}
\hline & $s_{1 / 2}$ & $p_{1 / 2}$ & $p_{3 / 2}$ & $d_{3 / 2}$ & $d_{5 / 2}$ & $f_{5 / 2}$ & $f_{7 / 2}$ \\
\hline \hline Number of basis & 35 & 32 & 32 & 25 & 25 & 25 & 25 \\
$\alpha_{0}$ & 0.00625 & 0.00638 & 0.00638 & 0.00654 & 0.00654 & 0.00667 & 0.00667 \\
$\beta$ & 2.03 & 2.07 & 2.07 & 2.19 & 2.19 & 2.27 & 2.27 \\
\hline
\end{tabular}

Table 2: Value of magnetic dipole $(A)$ and electric quadrupole $(B)$ hyperfine constants in $\mathrm{MHz}$ for ${ }^{25} \mathrm{Mg}^{+}$. (T) stands for $\mathrm{CC}$ with perturbative partial triples.

\begin{tabular}{lllrrrrr}
\hline States & CCSD $(\mathrm{T})^{(a)}$ & & MBPT & LCCSD $(\mathrm{T})$ & $(\mathrm{L}) \operatorname{CCSD}(\mathrm{T})$ & Others $^{(d)}$ & Experiment $^{(e)}$ \\
\hline \hline & $\mathrm{A}$ & $\mathrm{B}$ & $\mathrm{A}$ & $\mathrm{A}^{(c)}$ & $\mathrm{A}^{(c)}$ & $\mathrm{A}$ & $\mathrm{A}$ \\
$3 s_{1 / 2}$ & 592.86 & & $602(8)^{(b)}$ & 590.73 & 597.45 & 597.6 & $596.2544(5)$ \\
& & & $602.46^{(c)}$ & & & & \\
$4 s_{1 / 2}$ & 162.32 & & $164.65^{(c)}$ & 161.79 & 163.34 & 163.4 & \\
$3 p_{1 / 2}$ & 101.70 & & $103.20^{(c)}$ & 100.69 & 102.40 & 103.4 & \\
$4 p_{1 / 2}$ & 33.83 & & $34.26^{(c)}$ & 33.51 & 34.05 & & \\
$3 p_{3 / 2}$ & 18.89 & 22.91 & $19.94^{(c)}$ & 18.95 & 19.02 & 19.29 & \\
$4 p_{3 / 2}$ & 6.21 & 7.48 & $6.54^{(c)}$ & 6.24 & 6.25 & & \\
$3 d_{3 / 2}$ & 1.17 & 1.26 & $1.16^{(c)}$ & 1.16 & 1.17 & 1.140 & \\
$4 d_{3 / 2}$ & 0.51 & 0.48 & $0.505^{(c)}$ & 0.51 & 0.51 & & \\
\hline \hline
\end{tabular}

Refs. : (a) : Present work, (b) [3], (c) Present work, $(d):[4],(e):[2]$

carried out by using second order RMBPT is in agreement with the result of the earlier calculation (see table 2) 3].

In table 2 the results calculated by Safronova et al 4 using linearised coupled cluster in singles doubles approximation (LCCSD) are given. We have performed LCCSD(T) calculations and the results are given in the same table. The reasons for the discrepancy between the two linearised coupled-cluster calculations seem to be the inclusion of partial triple excitations by us and the different choice of basis sets. From our present calculations it is possible to determine the contributions from the non-linear clusters. These contributions vary from $0.3 \%\left(3 s_{1 / 2}\right)$ to $0.8 \%\left(3 d_{3 / 2}\right)$ for the different states. We find that if we take the linear contributions of the $T$ amplitudes but the nonlinear contributions of the combined $T$ and $S$ amplitudes and then perform the calculation of the hyperfine constants $(\mathrm{Eq}$ (28) at the linear level (which we have named as $(\mathrm{L}) \operatorname{CCSD}(\mathrm{T}))$, the result $\left(A\left(3 s_{1 / 2}\right)=597.45 \mathrm{MHz}\right)$ is in excellent agreement with the experiment (error is $\sim 0.2 \%$ ) for the ground state. But theoretically this approach is not complete and hence a proper inclusion of the nonlinear effects is desirable. Table 4 shows the contribution of triples in the final property. Some partial triples effect are taken into account iteratively according to Eq. (27) and the $S$ amplitude thus contain some partial triples. The effect of triples in calculation of properties (Eq. 28) thus comes from the triples effect included in $S$ amplitude. Figure 1 shows some of the typical diagrams which give rise to the effect due to triples, where figure 1 and a correspond to the terms $\widehat{V S_{2}}$ and $\widehat{V T}_{2}$ respectively.

For the present calculations, the number of basis functions actually used are the following : $16 s_{1 / 2}$, $14 p\left(p_{1 / 2}\right.$ and $\left.p_{3 / 2}\right), 11 d\left(d_{3 / 2}\right.$ and $\left.d_{5 / 2}\right)$ and $10 f\left(f_{5 / 2}\right.$ and $\left.f_{7 / 2}\right)$. Excitations from all the core orbitals have been considered.

The important contributions to the magnetic dipole hyperfine constants for different states are given in table 5. In particular, we have analyzed the contributions from various many body effects and have demonstrated that the most important contributions come from core polarization and pair correlation effects. The largest contribution comes from $\bar{O}$. The next two largest contributions come from $\left(\bar{O} S_{1}+\right.$ $\left.S_{1}^{\dagger} \bar{O}\right)$ and $\left(\bar{O} S_{2}+S_{2}^{\dagger} \bar{O}\right)$ which correspond to the pair-correlation (PC) and core-polarization (CP) effects respectively. The contribution from the corresponding MBPT terms are listed in table 6 . Figures 2 and 3 represents the pair-correlation and core-polarization diagrams in MBPT and CC respectively. 
Table 3: Convergence of results for A (3s $1 / 2$ state) using different basis sets

\begin{tabular}{ccccccccc}
\hline & $s_{1 / 2}$ & $p_{1 / 2}$ & $p_{3 / 2}$ & $d_{3 / 2}$ & $d_{5 / 2}$ & $f_{5 / 2}$ & $f_{7 / 2}$ & $A($ in $\mathrm{MHz})$ \\
\hline \hline Number & 11 & 9 & 9 & 8 & 8 & 7 & 7 & 562.10 \\
of basis & 14 & 12 & 12 & 10 & 10 & 8 & 8 & 589.18 \\
& 16 & 14 & 14 & 11 & 11 & 10 & 10 & 592.86 \\
& 17 & 15 & 15 & 11 & 11 & 10 & 10 & 592.86 \\
\hline \hline
\end{tabular}

Table 4: Comparative tables for the value of magnetic dipole $(A)$ hyperfine constants in $\mathrm{MHz}$ for ${ }^{25} \mathrm{Mg}^{+}$ calculated using CCSD and CCSD $(\mathrm{T})$. The difference between the values demonstrate the effect of triples.

\begin{tabular}{clll}
\hline States & CCSD(T) & CCSD & Experiment \\
\hline $3 s_{1 / 2}$ & 592.86 & 593.01 & $596.2544(5)$ \\
$4 s_{1 / 2}$ & 162.32 & 162.39 & \\
\hline \hline
\end{tabular}

Table 5: Comparative study of the contribution from different terms $(\operatorname{CCSD}(\mathrm{T}))$ containing the dressed operator $\bar{O}$ in determining the value of magnetic dipole hyperfine constant $A$ for ${ }^{25} \mathrm{Mg}^{+}$for the different states.

\begin{tabular}{ccccccccc}
\hline Terms & $3 s_{1 / 2}$ & $4 s_{1 / 2}$ & $3 p_{1 / 2}$ & $4 p_{1 / 2}$ & $3 p_{3 / 2}$ & $4 p_{3 / 2}$ & $3 d_{3 / 2}$ & $4 d_{3 / 2}$ \\
\hline \hline $\bar{O}$ & 468.819 & 131.616 & 77.975 & 26.400 & 15.337 & 5.196 & 1.262 & 0.563 \\
$\bar{O} S_{1}+S_{1}^{\dagger} \bar{O}$ & 40.046 & 8.256 & 7.344 & 2.096 & 1.442 & 0.412 & 0.070 & 0.031 \\
$\bar{O} S_{2}+S_{2}^{\dagger} \bar{O}$ & 77.002 & 20.587 & 14.891 & 4.872 & 1.832 & 0.519 & -0.175 & -0.086 \\
$S_{1}^{\dagger} \bar{O} S_{1}$ & 0.855 & 0.129 & 0.179 & 0.044 & 0.035 & 0.009 & 0.002 & 0.0007 \\
$S_{2}^{\dagger} \bar{O} S_{1}$ & 1.175 & 0.162 & 0.238 & 0.048 & 0.0004 & -0.007 & -0.003 & -0.001 \\
$S_{1}^{\dagger} \bar{O} S_{2}$ & 1.175 & 0.162 & 0.238 & 0.048 & 0.0004 & -0.007 & -0.003 & -0.001 \\
$S_{2}^{\dagger} \bar{O} S_{2}$ & 5.447 & 1.613 & 0.997 & 0.350 & 0.268 & 0.101 & 0.015 & 0.008 \\
\hline
\end{tabular}

Table 6: Comparative study of the most contributing terms containing the operator $O$ from $(\mathrm{CCSD}(\mathrm{T}))$ in determining the value of magnetic dipole hyperfine constant $A$ for ${ }^{25} \mathrm{Mg}^{+}$for the different states.

\begin{tabular}{ccccccccc}
\hline Terms & $3 s_{1 / 2}$ & $4 s_{1 / 2}$ & $3 p_{1 / 2}$ & $4 p_{1 / 2}$ & $3 p_{3 / 2}$ & $4 p_{3 / 2}$ & $3 d_{3 / 2}$ & $4 d_{3 / 2}$ \\
\hline \hline$O$ & 468.819 & 130.616 & 77.975 & 26.400 & 15.337 & 5.196 & 1.262 & 0.563 \\
$O S_{1}+S_{1}^{\dagger} O$ & 39.713 & 8.233 & 7.293 & 2.093 & 1.440 & 0.414 & 0.070 & 0.031 \\
$O S_{2}+S_{2}^{\dagger} O$ & 77.767 & 20.836 & 15.153 & 4.973 & 1.984 & 0.569 & -0.194 & -0.096 \\
\hline
\end{tabular}


Table 7: Individual contribution from the $O S_{1}$ and $O S_{2}$ diagrams for $3 s_{1 / 2}$ state. The values given correspond to the respective terms in $\mathrm{MHz}$.

\begin{tabular}{ccccc}
\hline Orbital & $\bar{O} S_{1}$ & $O S_{1}$ & $\bar{O} S_{2}$ & $O S_{2}$ \\
\hline \hline $3 s_{1 / 2}$ & 20.023 & 19.857 & & \\
& & & & \\
$1 s_{1 / 2}$ & & & 13.309 & 13.196 \\
$2 s_{1 / 2}$ & & & 26.665 & 25.699 \\
$2 p_{1 / 2}$ & & & 0.882 & 0.867 \\
$2 p_{3 / 2}$ & & & -0.958 & -0.878 \\
\hline \hline
\end{tabular}

We have listed the contributions from the different terms containing the dressed operator $\bar{O}$ in table [5 and table 6 gives the contributions from the terms containing the operator $O$ directly. The results given in tables [ 5 and 6 show that the $\mathrm{CP}$ contribution is larger than the $\mathrm{PC}$ in magnitude for all the states, although the ratio of the two effects is not uniform. It is important to note that the former contribution includes the hyperfine interaction of all the core orbitals while only a specific valence orbital is involved in this interaction for the latter (see Figure 3). These individual contributions are presented in table 7 for the ground state of $\mathrm{Mg}^{+}$. However, the ground state hyperfine constant $A$ for $\mathrm{Ba}^{+}$exhibits exactly the opposite behaviour [16]. Even though $\mathrm{Ba}^{+}$has more core electrons than $\mathrm{Mg}^{+}$, the relativistic enhancement of the valence $(6 s)$ magnetic dipole hyperfine interaction results in the value of $\mathrm{PC}$ exceeding that of CP.

\section{Conclusion}

In this paper we have carried out ab-initio relativistic coupled cluster calculations of magnetic dipole $(A)$ and electric quadrupole $(B)$ hyperfine constants for the ground and some excited states of ${ }^{25} \mathrm{Mg}^{+}$. Some contributions from partial triples are also taken into account in our calculation. We have shown that the dominant many-body contributions to these properties come from core-polarization and pair-correlation effects.

In addition to comparing with the available experimental data we have also predicted the values of $A$ and $B$ for a few states which could be of interest in the future. Using ion-trapping and other experimental techniques, it may be possible to measure both the magnetic dipole and electric quadrupole hyperfine constants for different states of ${ }^{25} \mathrm{Mg}^{+}$, thereby checking the accuracy of our calculations. This would constitute an useful test of the validity of the coupled-cluster theory in capturing the many-body effects in hyperfine interactions in light atomic systems with a single valence electron.

Acknowledgments : This work was supported by the BRNS for project no. 2002/37/12/BRNS.

The computation were carried out on our group's 4 CPU E450 Sun Ultra SPARC machine in IIA and on CDAC, Bangalore's Teraflop Supercomputer Param Padma.

\section{References}

[1] E. Arimondo, M. Inguscio and P. Violino, Rev. Mod. Phys, 49, 31 (1977).

[2] M. Itano and D. J. Wineland, Phys. Rev. A, 24, 1364 (1981).

[3] S. Ahmed, J. Andriessen and T. P. Das, Phys. Rev. A, 27, 2790(1983).

[4] M. S. Safronova, W. R. Johnson and A. Derevianko, Phys. Rev. A, 58, 1016 (1998).

[5] P. Jönsson, A Ynnerman, C Froese Fischer, M.R. Godefroid and J. Olsen, Phys. Rev. A, 53, 4021 (1996).

[6] A. C. Melissinos and J. Napolitano, Experiments is Modern Physics, 2 ed., p 215 (Academic Press, London), 2003

[7] M. Bhattacharya, C. Haimberger and N. P. Bigelow, physics/0304031. 
[8] I. Lindgren and J. Morrison, Atomic Many-Body Theory (Springer, Berlin) 1985.

[9] K. T. Cheng and W. J. Child, Phys. Rev. A, 31, 2775 (1985).

[10] I. Lindgren and A. Rosen, Case Stud. At. Phys., 4, 197 (1974).

[11] R. J. Bartlett, Modern Electronic Structure Theory, vol-II, p.1047, Ed. D. R. Yarkony (World Scientific, Singapore)1995.

[12] K. Raghavachari, G. W. Trucks, J. A. Pople and M. Head-Gordon, Chem. Phys. Lett., 157, 479 (1989).

[13] G. Gopakumar, H. Merlitz, R. K. Chaudhuri, B. P. Das, U. S. Mahapatra, and D. Mukherjee, Phys. Rev. A, 66, 032505 (2002).

[14] R. K. Chaudhuri, P. K. Panda and B. P. Das, Phys. Rev. A, 59, 1187 (1999).

[15] R. C. Raffenetti and K. Ruedenberg, J. Chem. Phys, 59, 5978 (1973).

[16] B. K. Sahoo, G. Gopakumar, R. K. Chaudhuri, B. P. Das, H. Merlitz, U. S. Mahapatra, and D. Mukherjee, Phys. Rev. A 68, 040501 (R) (2003). 